\title{
Research on the Straightening Stroke Prediction Precision of Guide Hong $\mathrm{Lu}^{1,2, \mathrm{a}}$, Sheng $\mathrm{GaO}^{1, \mathrm{~b}^{\star}}$, Jianwen Chen ${ }^{1, \mathrm{c}}$, Xiong Xiao ${ }^{1, \mathrm{~d}}$, Fengren $\mathrm{Yi}^{3, \mathrm{e}}$ \\ ${ }^{1}$ School of Mechanical and Electronic Engineering, Wuhan University of Technology, Wuhan 430070, China \\ ${ }^{2}$ Key Laboratory of Hubei Province for Digital Manufacture, Wuhan 430070, China \\ ${ }^{3}$ Texas A\&M University, College Station 77840, U.S.

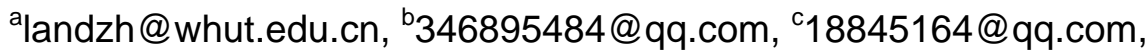 \\ d740312971@qq.com, èyifengr@yahoo.com
}

Key Words: Linear Guide; Straightening; Stroke Prediction Model; Strain Measure

Abstract. In order to improve straightening precision and efficiency, theory analysis and experiment is adopted to build straightening stroke prediction model. A theoretical prediction model is built and analyzed based on elastic-plastic theory. Then, a strain measuring scheme is proposed based on wireless sensing technology, which can detect strain of guide online. Another prediction model is established depend on experiment data. A proving experiment is conducted to compare three stroke prediction model built by elastic-plastic theory, Finite Element Analysis (FEA) and strain measuring experiment. Finally, a predicted model with minimal error was come up with for improving straightening precision.

\section{Introduction}

With the rapid development of industrial automation, precision linear guide is widely used in industrial equipment and its straightness directly determines the quality of the product [1]. The process of rolling, forging, extrusion, cooling will cause the bending deformation of the linear guide. Therefore, it is necessary to carry on the straightening to ensure the straightness. Since the end of last century 90's, developed countries such as the United States, Germany, and Japan have researched the straightening mechanism and equipment [2]. Then, theory and experiment studies about straightening have been carried out in China [3]. But at present, the research on the prediction method and the control strategy of the straightening stroke has restricted the development of the precision straightening technology. In this paper, by using different methods to establish stroke prediction model of LG linear guide and combining with precision straightening experiment, a new method which can improve the precision and efficiency of the straightening stroke prediction is proposed.

\section{Straightening theory and theoretical prediction model of straightening stroke}

Straightening theory. The straightening method used in this paper is called the three point bending [4]; the schematic diagram is shown on Fig.1. The guide that is non-compliance is transported into straightening machine by roller. Then, guide deflection, $\delta_{0}$, is detected automatically which will be converted to stroke and control the indenter press guide. The reverse bending deflection of guide is $\delta_{\mathrm{b}}$. After unloading, part of guide rebounds and the springback value is $\delta_{\mathrm{r}}$. If $\delta_{\mathrm{b}}=\delta_{\mathrm{r}}$, output the guide. Otherwise, repeat the above steps until the guide straightness is up to the standard [5].

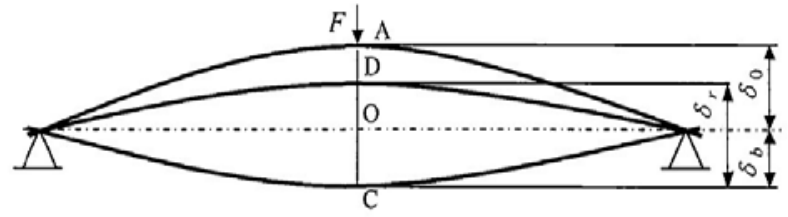

Fig. 1 principle diagram of straightening machine

Theoretical prediction model. The research of theoretical model is mainly focused on the following three aspects [6]: (1) Bending theoretical model based on the straightening curvature; (2) 
Bending theoretical model based on load and deflection; (3) Elastic-plastic analysis model of the straightening process deflection. The theory of these models is mature, but application is difficult.

For LG guide, the relationship between the load and deflection during the elastic-plastic deformation stage is shown on [7]:

$$
\left\{\begin{array}{l}
\mathrm{S}=\frac{M_{t}}{3 E I} \frac{L^{2}}{\bar{M}^{2}}[5-(3+\bar{M}) \sqrt{3-2 \bar{M}}] \\
F=\frac{2 M_{t}}{L} \bar{M}
\end{array}\right.
$$

where $M_{t}$ is elastic limit moment, $E$ is elastic modulus, $I$ is inertia moment, $L$ is half of length, $B$ is breadth, $\mathrm{H}$ is height, $\sigma_{\mathrm{s}}$ is yield limit, $\bar{M}$ is ratio of current moment and elastic limit moment.

According to the material mechanics, $\mathrm{F}=\frac{6 E I}{L^{3}}(S-\delta), \mathrm{S}$ is straightening stroke, $\delta$ is deflection. Combining these equations, the theoretical prediction model of straightening stroke is expressed by

$$
S=\frac{M_{t}^{3} L^{6}}{27 E^{3} I^{3}(S-\delta)^{2}}\left\{5-\left[3+\frac{3 E I(S-\delta)}{M_{t} L^{2}}\right] \sqrt{3-\frac{6 E I(S-\delta)}{M_{t} L^{2}}}\right\}
$$

\section{The Prediction model of straightening stroke based on on-line strain detection experiment}

Experiment principle. The guide strain need to be detected on-line in straightening process, so it's necessary to choose a measure system that can collect, transmit and record strain data in real time. Wireless network sensor technology is used in the experiment that can transmit the collected data to the information processing center by the optimization of the radio waves [8], and make the data collecting is real-time and efficient.

This experiment cannot directly obtain the guide deflection, needs establish a relation between strain and deflection. Straightening process is an elastic-plastic deformation process. The relationship can only be built depended on geometrical characteristics of guide. According to the plane assumption of pure bending beam, the relationship between the curvature radius of neutral layer and deflection is expressed as:

$$
\left(\rho-\frac{H}{2}-\delta\right)^{2}+L^{2}=\left(\rho-\frac{H}{2}\right)^{2}
$$

Intercepting a micro segment at the midpoint of the guide, according to material mechanics, the relation between the strain of guide surface and the curvature radius of neutral layer is expressed by:

$$
\varepsilon=\frac{H / 2}{\rho}
$$

Uniting the eq. 3 and eq.4, the function about strain and deflection is given by:

$$
\delta=\frac{\left(\frac{1}{\varepsilon}-1\right) H \pm \sqrt{\left(\frac{1}{\varepsilon}-1\right)^{2} H^{2}-4 L^{2}}}{2}
$$

Where $\rho$ is the curvature radius of neutral layer, $\varepsilon$ is the strain of guide surface.

Experiment scheme. Choose the wireless strain node SG402 and paste strain gauges on both sides of the guide, which make up Wheatstone full bridge circuit and connect with wireless strain node. In order to ensure the accuracy of measuring, strain gauges should be arranged in the middle of guide. However, the indenter directly touches the middle of the guide, and the strain gauge in the contact area will be destroyed. Therefore, the final distance between strain gauge and the guide midpoint is $35 \mathrm{~mm}$ depended on the indenter size. The scheme ensures both the normal work of strain gauge and the accuracy of the experimental data. The layout of the experiment scheme and the data transmission diagram are shown in Fig.2 and the experiment practicality is shown in Fig.3. 


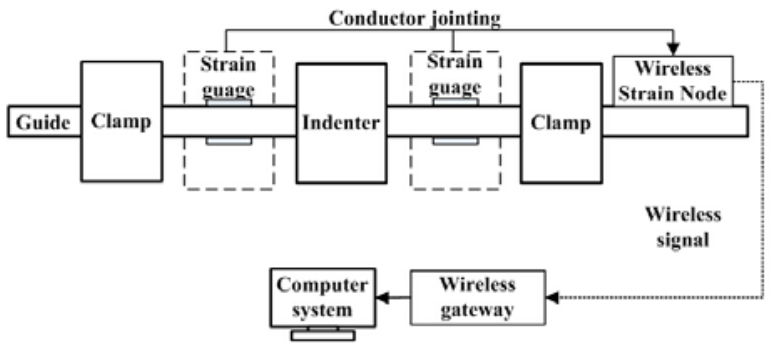

Fig. 2 experimental plan layout

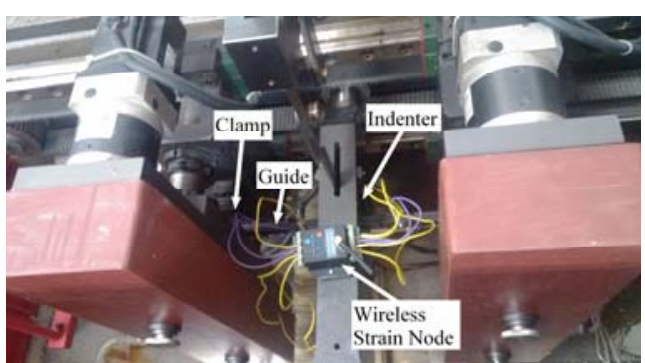

Fig.3 experiment practicality

Straightening stroke prediction model based on strain experiment. The basic parameters of the experiment guide are as follows: $\mathrm{E}=190088.5 \mathrm{MPa}, 2 \mathrm{~L}=300 \mathrm{~mm}, \mathrm{~B}=15.5 \mathrm{~mm}, \mathrm{H}=14.5 \mathrm{~mm}$, $\sigma_{\mathrm{s}}=382 \mathrm{MPa}$. Using eq.5 to calculate the deflection variation of each straightening step based on experiment data. The cubic polynomial fitting is applied to get the straightening stroke prediction model based on experiment data. In addition, there are other two models. One is the above theoretical prediction model, the other one is the FEA prediction model [5]. As shown on Fig.4.

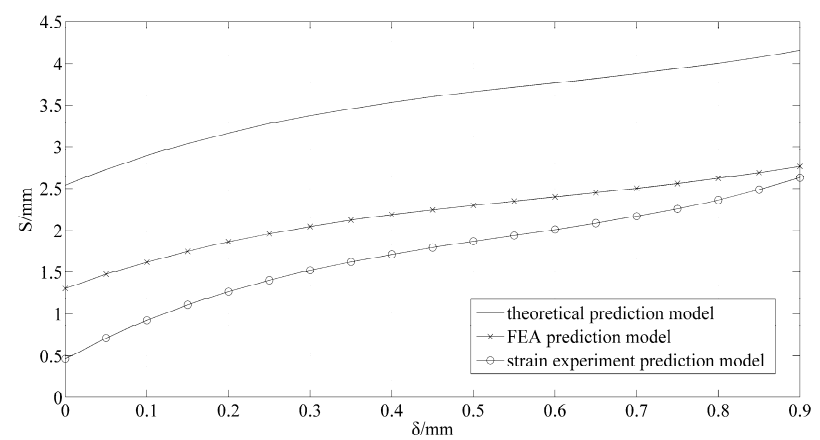

Fig. 4 three stroke prediction models

Fig. 4 shows that the overall trend of the three models is basically the same from 0 to $0.9 \mathrm{~mm}$, however, the curve of theoretical prediction model is much higher than the other two models. When the initial deflection is less, the curve of FEA prediction model is slightly higher than the strain experiment prediction model. But the difference of the predicted value of these two models is small. Analysis precision of straightening stroke prediction model. In order to compare the accuracy of these three models, the follow experiment has been carried out. In this experiment, the three-coordinate measuring instrument made by Brown\&Sharpe is applied, which can measure the three coordinates of the points on the guide surface, and calculate the deflection of guide automatically and precisely. Choosing three guides with the same material and using the above instrument to obtain the initial deflection of guide. Then predict straightening stroke depended on the three models above and compare with the straightening stroke. The result is shown on Table 1.

Table 1 predicted and actual straightening stroke

\begin{tabular}{ccccc}
\hline $\begin{array}{c}\text { Initial } \\
\text { deflection[mm] }\end{array}$ & $\begin{array}{c}\text { Actual } \\
\text { straightening } \\
\text { stroke[mm] }\end{array}$ & $\begin{array}{c}\text { theoretical } \\
\text { prediction } \\
\text { model[mm] }\end{array}$ & $\begin{array}{c}\text { FEA prediction } \\
\text { model [mm] }\end{array}$ & $\begin{array}{c}\text { strain experiment } \\
\text { prediction } \\
\text { model[mm] }\end{array}$ \\
0.104 & 1.055 & 2.923 & 1.631 & 0.956 \\
0.518 & 2.139 & 3.714 & 2.389 & 1.905 \\
0.876 & 2.826 & 4.186 & 2.642 & 2.574 \\
\hline
\end{tabular}

Table 1 shows the error of theoretical prediction model is largest. The main reason is that the material is simplified as an ideal elastic-plastic material in the deduction process. In the other two models, the error of the prediction model based on the strain experiment is smaller. But in the third experiment, its prediction error is slightly larger than the FEA prediction model. It is because the deflection deformation is small in the strain measure experiment; there is few experiment data when deflection is large. However, in general, the precision of the stroke prediction model based on strain measure experiment is the highest in these three models. 


\section{Conclusions}

(1) As the control variable of straightening, straightening stroke is more convenient than other variable. It can be precisely controlled and help to improve the straightening precision.

(2) The strain measure experiment scheme realizes the on-line measuring, which make experiment data can reflect the straightening state of guide in real time. So stroke prediction model based on this experiment is the most precise.

(3) For different guide, it is necessary to use different prediction model to control straightening process. The material properties of work piece and experimental data processing method should be further researched in the future to establish prediction model that can adapt to a wider range.

\section{Acknowledgements}

This work was financially supported by the National Natural Science Foundation of China (51275372), the Fundamental Research Funds for the Central Universities (155204002).

\section{References}

[1] Jianhua Wang, Tongmin Ma and Zhifeng Liu. Journal of Chemical \& Pharmaceutical Research Vol. 6(2014), p. 2414-2421.

[2] B. John. Iron Steel Eng Vol.11 (1997), p. 38-45.

[3]Aiwen Cao, Guoliang Xiong. China Metal Forming Equipment \& Manufacturing Technology Vol. 1(2007), p.9-12(In Chinese).

[4] T.N. Das, A. Singh. Journal of Engineering for Industry Vol. 113(1991), p. 224-227.

[5] Hong Lu, He Ling and J. Leopold. Sci. China Ser. E: Tech. Sci. Vol. 52(2009), p. 1866-1873.

[6]Ben Guan, Yong Zang and Weizhuang Qu. Machinery Design \& Manufacture Vol. 49(2012), p. 266-268(In Chinese).

[7]Fu Cui: Straightening theory and machine (Metallurgical Industry Press, Beijing 2002) (In Chinese).

[8] D.I. Ozlem, V.H. Lodewijk and J. Pierre. Ad Hoc Networks Vol. 9(2011), p. 73-94. 